REVIEW ARTICLE

\title{
Clinical Progress of Toremifene and Tamoxifen in Endocrine Ther- apy for Breast Cancer
}

\author{
Zhu Lizhe, Li Xiongxiong, Yu Shuyao and Ren Yu*
}

Department of Breast Surgery, The First Affiliated Hospital of Xi'an Jiaotong University, China

*Corresponding author: Ren Yu, Department of Breast Surgery, The First Affiliated Hospital of Xi'an Jiaotong University, Xi'an 710061, China, E-mail: renyyyy@126.com

\begin{abstract}
Researches on the breast cancer pathogenesis have been greatly progressed within the past few decades, so does the immunohistochemical classification of breast cancer. Recent clinical trials and studies have shown that endocrine therapy has become a clear target treatment with confirmed efficacy of breast cancer. In the drug selection of endocrine therapy for breast cancer, tamoxifen and toremifene have been approved by the U.S. Food and Drug Administration (FDA) successively on the treatment of peri-menopausal breast cancer patients with positive hormone receptor. A number of domestic and foreign researches have been done in comparison of using toremifene and tamoxifen alone, or both in the endocrine therapy for breast cancer; however results and conclusions vary among different studies. This article will mainly focus on reviewing literatures of this field from four aspects, the efficacy, safety, quality of life and economic benefits, and discussing the clinical progress of toremifene and tamoxifen in endocrine therapy for breast cancer.
\end{abstract}

\section{Keywords}

Breast neoplasms, Toremifene, Tamoxifen, Endocrine therapy, Validation studies

\section{Introduction}

Worldwide, breast cancer is one of the top malignant tumors in women. According to the statistic of WHO, each year, there are nearly 1.2 million people suffering from it, and approximately 0.5 million women dying from it. Its incidence is increasing by $2 \%-8 \%$ per year. In China, over one-third of female patients are in premenopausal status while getting confirmed diagnosis, among which $60 \%-70 \%$ of patients are positive for estrogen or progesterone receptor [1]. Therefore, most patients need endocrine therapy.
In the endocrine therapy of breast cancer, there are two kinds of drugs which are commonly used, tamoxifen and toremifene. Tamoxifen had been approved by the U.S. Food and Drug Administration as a kind of selective estrogen receptor modulators as early as 1977. It was used in the treatment of advanced breast cancer with hormone receptor positive, nevertheless as the first generation of selective estrogen receptor modulators, TAM produced antagonistic action of estrogen in breast tissue. Meanwhile, estrogenic activity was also produced in the uterus and bone, thus caused other adverse events like genital lesions (for example, the increasing risk of endometrial cancer) at the same time while treating breast cancer. Toremifene was a kind of estrogen receptor regulator which was approved by the FDA for the treatment of breast cancer after TAM. It had been used in the clinical adjuvant therapy on breast cancer since 1995. Recently, a number of domestic and foreign studies have discussed the similar efficacy and adverse reactions of TOR and TAM in the treatment of breast cancer in various stages. This article will summarize relevant literature and data from four aspects, the efficacy, safety, quality of life and economic benefits, and to discuss the clinical progress of toremifene and tamoxifen in endocrine therapy for breast cancer.

\section{Comparative study of TOR and TAM in the endo- crine therapy of breast cancer}

Comparative study of efficacy: Comparative study of endocrine therapy for early stage breast cancer: In the studies of endocrine therapy for early breast cancer, researches are not only targeting on large data sample,

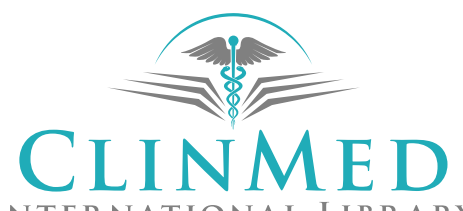

INTERNATIONAL LIBRARY

Citation: Lizhe Z, Xiongxiong L, Shuyao Y, Yu R (2017) Clinical Progress of Toremifene and Tamoxifen in Endocrine Therapy for Breast Cancer. Int J Surg Res Pract 4:058. doi.org/10.23937/2378$3397 / 1410058$

Received: November 09, 2017: Accepted: December 04, 2017; Published: December 06, 2017

Copyright: (C) 2017 Lizhe Z, et al. This is an open-access article distributed under the terms of the Creative Commons Attribution License, which permits unrestricted use, distribution, and reproduction in any medium, provided the original author and source are credited. 
but also on analyzing small data sample. Zhang Lin, et al. [2], collected 236 cases of breast cancer patients who were treated with endocrine drugs after taking surgery. All patients were divided into TAM 115 and TOR 121 randomly. Statistical analysis had found that TOR was better than TAM at both the 2 year survival rate and the disease free survival. Although there were no statistical significance between the two Qin Tao, et al. [3] arranged 247 cases clinical pathological data of postmenopausal breast cancer patients with Luminal type. Results showed that 6 year Disease Free Survival (DFS) of TOR and TAM were $77.0 \%$ and $79.2 \%$ respectively, and 6 year overall survival (OS) was $88.4 \%$ and $87.4 \%$ respectively. However, there was no significant difference between the two groups (OS: HR $=0.794, P=0.589$; DFS $\mathrm{HR}=1.132, P=0.686)$. Kimura, et al. [4] took Phase 3 randomized clinical trials in 252 postmenopausal early breast cancer patients in Japan. The study analysis showed that 5 year survival rate were roughly equivalent (TOR 97.0\%, TAM 96.9\%). Ma Dachang, et al. [5] led on a larger sample research. It totally enrolled 3084 patients in 3 randomized controlled trials. The Meta analysis reflected that TOR did not improve patients overall survival (OS) $(\mathrm{RR}=1.01,95 \% \mathrm{Cl}$ : 0.98 1.03, $P=$ $0.560)$ and Disease Free Survival (DFS) (RR $=1.01,95 \%$ Cl: 0.98 1.04, $P=0.480$ ) compared to TAM. Chi, et al. [6] selected 7242 patients for a randomized controlled trial and only 3747 patients had been observed for five years long. Results came from large amount of samples reflected that, as for early breast cancer, TOR had a longer 5 year survival rate compared with TAM $(O R=1.25$, 95\% Cl: 1.04 1.50).

Collectively, these researches showed a comparison

Table 1: The characteristic of studies- Overall Survival (OS).

\begin{tabular}{|c|c|c|c|c|c|}
\hline \multirow{3}{*}{ Study } & \multicolumn{4}{|c|}{ Patient number } & \multirow{3}{*}{ Follow-up } \\
\hline & \multicolumn{2}{|c|}{ TOR } & \multicolumn{2}{|l|}{ TAM } & \\
\hline & Events & Total & Events & Total & \\
\hline Zhang 2010 & 121 & 121 & 115 & 115 & 2 years \\
\hline Kimura 2014 & 122 & 126 & 123 & 126 & 5 years \\
\hline Qin 2013 & 58 & 66 & 158 & 181 & 6 years \\
\hline Ma 2012 & 1386 & 1552 & 1359 & 1532 & unavailable \\
\hline Chi 2013 & 1618 & 1890 & 1543 & 1857 & 5 years \\
\hline
\end{tabular}

of TOR and TAM on the overall survival (OS). Table 1 shows the characteristic of studies. From the Figure 1 , we can see that the $P$ value is 0.05 . It means that although TOR has a better overall survival, there are no statistical significance between the two (at the standard of $P=0.05$ ). Meanwhile, only three researches have accurate statistics data of DFS. The Table 2 shows the characteristic of them. The Figure 2 reflects that the DFS of TOR is similar to the DFS of TAM.

1. Comparative study of endocrine therapy for advanced breast cancer: Compared with the effectiveness of endocrine therapy in early breast cancer, researches and samples on advanced breast cancer are rare. Lu Qiang, et al. [7] selected a total of 58 cases of recurrent or metastatic female breast cancer patients in his hospital. They detected those patients' liver function and blood lipid by vein blood before endocrine therapy. After 12 months' treatment, the results showed that the effective rate of TAM and TOR were $62.1 \%$ and $69.0 \%$ respectively. There was no significant difference between the two groups. Zhou, et al. [8] did heterogeneity statistical analysis. Results suggested that there existed no significant difference in overall survival rate and DFS between TOR and TAM with breast cancer. Ye, et al. [9] collected 9 randomized controlled trials with a total of 4768 cases (including TOR 2,587, TAM 2,181) for Meta analysis. Results reflected that in the Objective Response Rate (ORR), Time to Progression (TTP) and Overall Survival (OS), both the two had similar efficacy. Ma Wen, et al. [10] selected 10 randomized controlled trials which involved 3,680 patients with advanced breast cancer. This study showed that the differences between the two were not statistically significant on the OS and DFS at the first year and the third year, but they

Table 2: The characteristic of studies- Disease Free Survival (DFS).

\begin{tabular}{|l|l|l|l|l|l|}
\hline \multirow{4}{*}{ Study } & \multicolumn{4}{|l|}{ Patient number } & \multirow{2}{*}{ Follow-up } \\
\cline { 2 - 6 } & TOR & & TAM & & Follo \\
& Events & Total & Events & Total & \\
\hline Zhang 2010 & 120 & 121 & 114 & 115 & 2 years \\
\hline Qin 2013 & 51 & 66 & 143 & 181 & 6 years \\
\hline Ma 2012 & 1307 & 1552 & 1278 & 1532 & unavailable \\
\hline
\end{tabular}

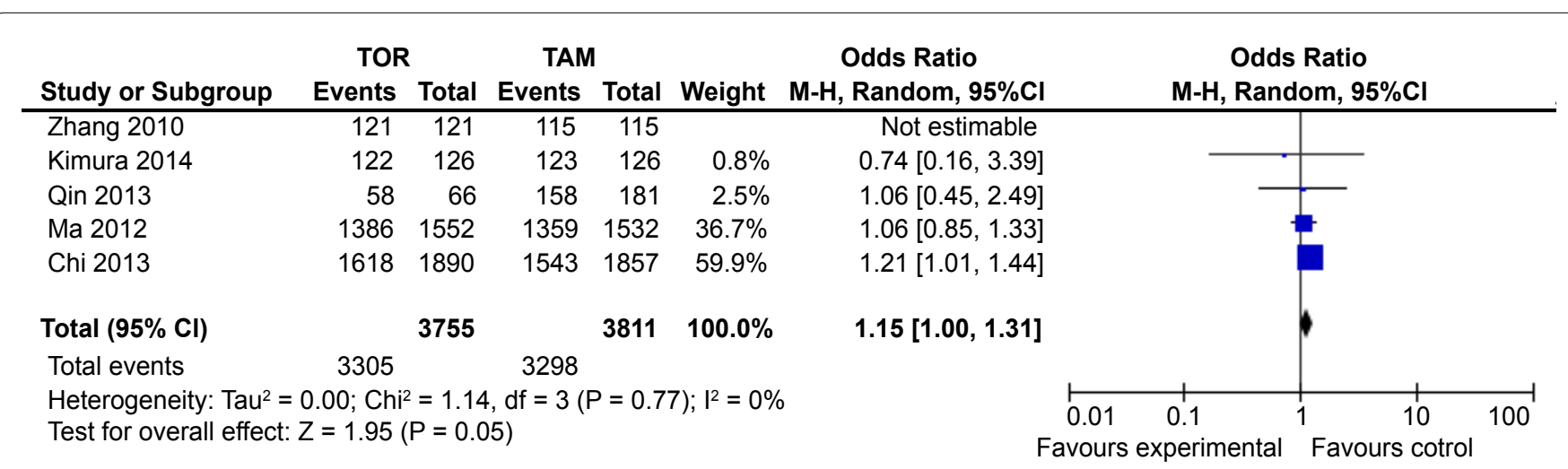

Figure 1: The forest plot of OS for TOR versus TAM in the treatment of breast cancer. 


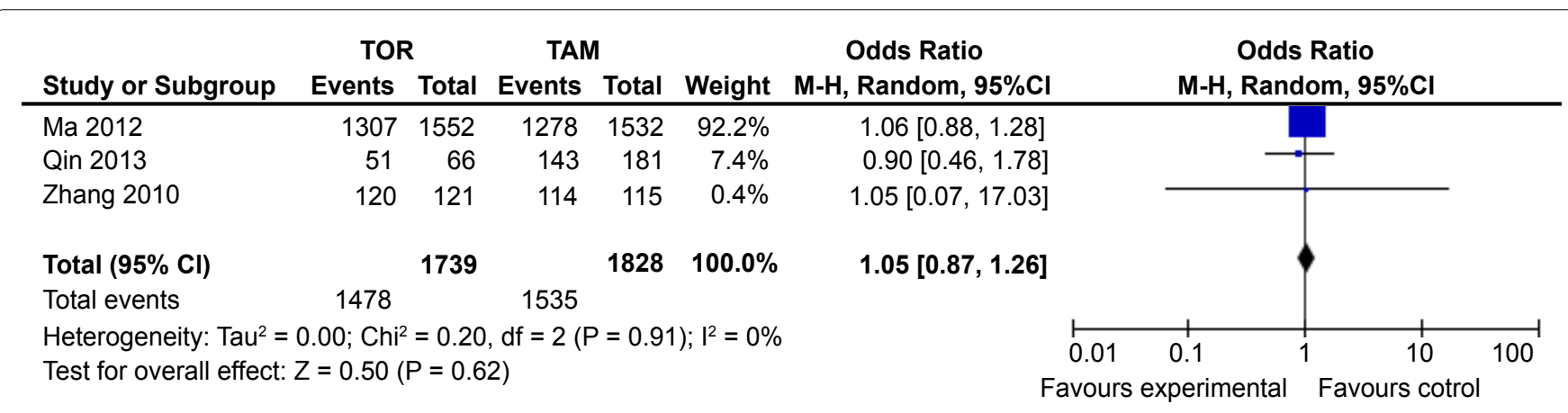

Figure 2: The forest plot of DFS for TOR versus TAM in the treatment of breast cancer.

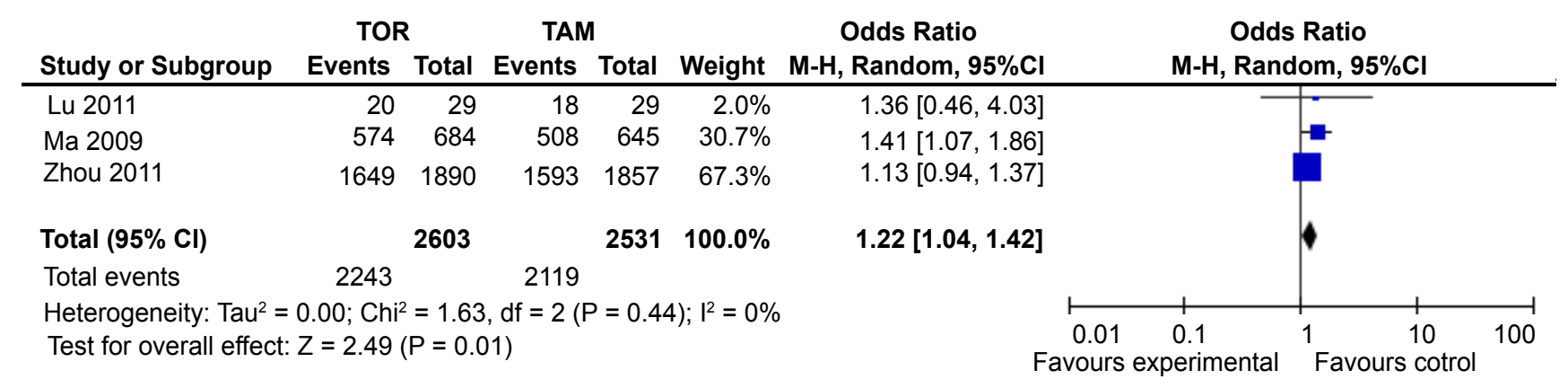

Figure 3: The forest plot of OS for TOR versus TAM in the treatment of breast cancer.

Table 3: The characteristic of studies- Overall Survival (OS).

\begin{tabular}{|c|c|c|c|c|c|}
\hline \multirow{3}{*}{ Study } & \multicolumn{4}{|c|}{ Patient number } & \multirow{3}{*}{ Follow-up } \\
\hline & \multicolumn{2}{|c|}{ TOR } & \multicolumn{2}{|l|}{ TAM } & \\
\hline & Events & Total & Events & Total & \\
\hline Lu 2011 & 20 & 29 & 18 & 29 & 1 years \\
\hline Zhou 2011 & 1649 & 1890 & 1593 & 1857 & $3-5$ years \\
\hline Ma 2009 & 574 & 684 & 508 & 645 & 5 years \\
\hline
\end{tabular}

had statistically significant in the fifth year on OS (RR $=1.06,95 \% \mathrm{Cl}: 1.01 \sim 1.12)$ and DFS (RR $=1.08,95 \% \mathrm{Cl}$ : 1.02 1.15). This showed that TOR was better than TAM in the long term. Table 3 shows the characteristic of the three studies (1. The study of Ye, et al. [9] is not taken into analysis because the data is not available in the article. 2. For the study of Ma Wen, et al. [10], we only take the group which have 5 years follow-up into analysis). We do a forest plot using data from above studies. It can be seen in the Figure 3. The test for overall effect shows that the $P$ value is 0.01 . It means that TOR has a better overall survival than TAM in the longer term and this difference has statistically significant.

Comparative study of safety: At present, answer of the question "Which one has a higher safety?" regarding the application of TAM or TOR in the adjuvant endocrine therapy for breast cancer is still controversial. There are two major ideas- either both two drugs have similar adverse reactions or the opposite one.

One faction believes that both two drugs have similar adverse reactions. Kimura, et al. [4] took Phase 3 randomized clinical trials in 252 postmenopausal early breast cancer patients in Japan. Results showed that the adverse reactions were similar; most of them were mild to moderate adverse reactions. Zhou, et al. [8] had done statistically analysis on 4 randomized controlled clinical trials of totally 3,747 cases (including TOR 1,890, TAM $1,857)$. The results reflected that the probability of getting endometrial polyps and endometrial cancer was the same in two groups, and the differences between those two drugs also existed no statistically significance on thrombotic events including deep vein thrombosis (OR $\left.=0.68,95 \% \mathrm{Cl}: 0.40^{\sim 1.17}, P=0.926\right)$, Cerebrovascular accident (OR $=0.59,95 \% \mathrm{Cl}: 0.32 \sim 1.09, P=0.438$ ), and pulmonary embolism (OR $=0.91,95 \% \mathrm{Cl}: 0.42 \sim 2.01, P=$ $0.618)$. Meta analysis proved that TOR could replace TAM for adjuvant endocrine therapy in perimenopausal and postmenopausal breast cancer effectively and safely.

Another faction of researchers holds the opinion that TOR is better than TAM and the difference of adverse reactions between the two is statistically significant. Lin Yan, et al. [11] divided 130 breast cancer patients into TAM group and TOR group according to different therapeutic regimen. The results displayed that the adverse reactions of TOR, especially severe adverse reactions were lower than TAM. Although both of them had an impact on the endometrium, the degree of TOR was slightly lower. Similarly, Study taken by Yan Han, et al. [12] showed that TOR had a lower incidence in some adverse events compared with TAM, such as incidence of ocular toxic and blood vessel embolism events. The evaluation results of the study showed that the safety of TOR was significantly higher than that of TAM in the occurrence of vascular embolism and ophthalmological adverse events. In addition, the occurrence rate of vaginal discharge in TOR was higher than that in TAM (OR = 
1.30, 95\% Cl: 1.04 1.63). Moreover, the incidence of endometrial thickening in patients treated with TOR was significantly lower than that of TAM $(P<0.05)$. At the present stage, there still exist some controversies about the gynecological safety. Also, more and more clinical trials are needed to prove it. Lu Qiang, et al. [7] did further study on changes of liver function and blood lipid induced by using the two drugs. They discovered that the incidence of liver function damage was similar, however, the Triglyceride (TG) was significantly lower while High Density Lipoprotein (HDL) was significantly higher in TOR group. Compared with TAM group, the difference was statistically significant $(P<0.05)$. It illustrated that TOR had a certain effect on ameliorating blood lipid. Compared with the above research, Ye, et al. [9] collected a large sample data of totally 4,768 cases (including TOR 2,587, TAM 2,181) which showed that the adverse events were similar in the two groups. Nevertheless, TOR could cause less vaginal bleeding ( 4.0 vs. $6.7 \%, \mathrm{P}=$ $0.01)$, headache (0.2 vs. $3.1 \%, P=0.02)$ and thrombotic events $(4.7$ vs. $7.0 \%, P=0.04)$. As for early breast cancer, compared to TAM, using TOR would cause leucorrhea increased $(O R=1.32,95 \%$ Cl: 1.01 1.73), Serum Triglyceride Content Decreased Significantly (SMD = -1.01 , 95\% Cl: -1.89 -0.14), Serum Low Density Lipoprotein Decreased Slightly (SMD $=0.45,95 \% \mathrm{Cl}: 0.07^{\sim} 0.84$ ), the Bone Density of Ward's Region Decreased (SMD = 0.36, 95\% Cl: -0.71 -0.01) and High Density Lipoprotein Significantly Increased (SMD $=0.43,95 \% \mathrm{Cl}: 0.08^{\sim} 0.77$ ). For advanced breast cancer, TOR would cause vaginal bleeding increased (OR $=0.45,95 \% \mathrm{Cl}: 0.26 \sim 0.80)$, and Serum Triglyceride Level Significantly Decreased (SMD $=-1.15,95 \%$ Cl: $-1.90^{\sim}-0.39$ ). We summarize the above studies. We can see that they have done researches on

Table 4: The characteristic of studies- Comparative study of safety.

\begin{tabular}{|l|l|l|l|l|}
\hline \multirow{4}{*}{ Study } & \multicolumn{3}{|l|}{ Patient number } \\
\cline { 2 - 5 } & TOR & & \multicolumn{2}{l|}{ TAM } \\
\cline { 2 - 5 } & Events $^{\mathbf{a}}$ & Total & Events $^{\text {a }}$ & Total \\
\hline Kimura 2014 & 16 & 126 & 14 & 126 \\
\hline Zhou 2011 & 36 & 1864 & 35 & 1845 \\
\hline Lin 2012 & 10 & 50 & 21 & 80 \\
\hline Yan 2014 & 130 & 1802 & 169 & 1759 \\
\hline
\end{tabular}

${ }^{a}$ Got adverse actions of drugs on gynecological disorders. different kinds of adverse reactions. Therefore, we take the most common one-gynecological disorders as a representative to do an analysis. Table 4 shows the characteristic of the selected studies. Consequently, Figure 4 reflects that TOR has lower adverse actions on gynecological disorders than TAM. The $P$ value is 0.03 . So it exists statistically significance at the standard of $P=0.05$.

Comparative study of quality of life: A number of domestic and foreign researches have not been unanimous on the quality of life after using TOR and TAM. Gershanovich, et al. [13] used ECOG scoring criteria for the evaluation of quality of life. They hold the opinion that TOR was superior to TAM in terms of quality of life (40.4\% vs. $21.6 \%)$. By contrast, Hayes, et al. [14] considered that there was no difference between the two groups in the quality of life (both the two groups were $7 \%$ ). Pagani, et al. [15] have found that the main reason affecting the quality of life is the body condition reduced, facial flushing, sweating and etc. But there was no significant difference between the two groups. At the same time, it was also believed that the above symptoms might be cause by chemotherapy drugs.

\section{TOR combined with TAM in the endocrine therapy of breast cancer}

For the moment, the treatment of advanced breast cancer are basically chemotherapy, chemotherapy combined with endocrine therapy, single use endocrine drugs or combined several endocrine drugs together. The study of Li Guangming, et al. [16] showed that TOR combined with TAM improved the recent effective treatment rate and health related quality of life compared with using NP program alone in the application of advanced breast cancer. Meanwhile, there was no change in the side effects compared with chemotherapy alone. Li Wenhua, et al. [17] had done a research that compared using TAM singularly and combining with other drugs. Result showed that there was no significant difference in liver function among all groups. Only Alkaline Phosphatase (ALP) was raised, however, it was in the range of normal values. From the study hold by Lan Ying, et al. [18], the data showed there were no statistically significance on ALP. Most of the studies include ALP as a part of liver

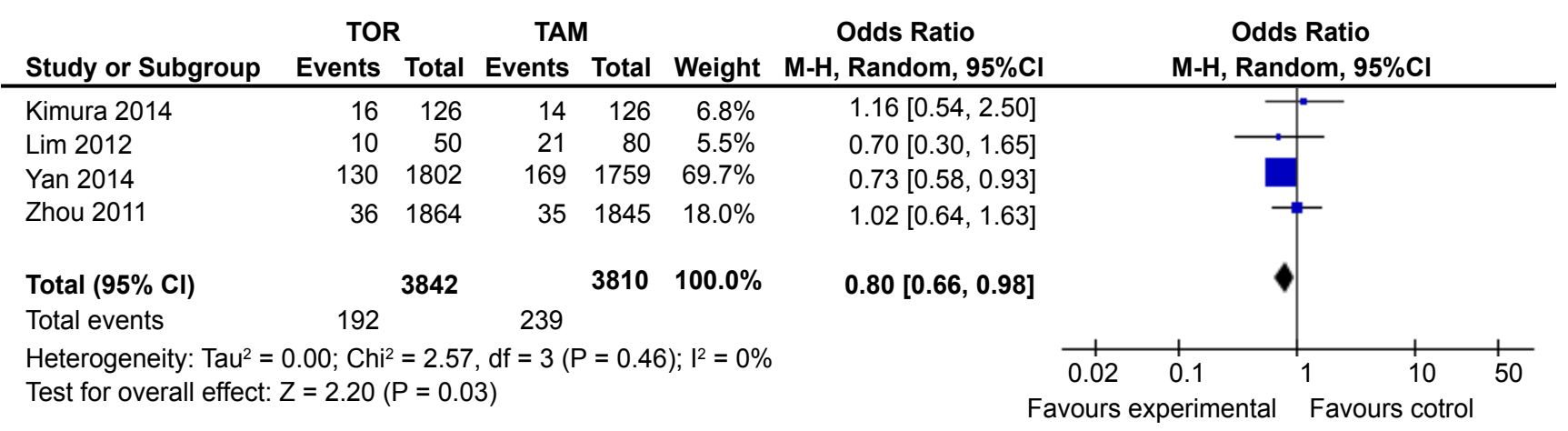

Figure 4: The forest plot of gynecological disorders for TOR versus TAM in the treatment of breast cancer. 
function evaluation, like ALT, AST, GGT, and et al. Nevertheless, the mechanism of increasing ALP is not clear, maybe relevant to the destruction of the liver function.

\section{Pharmacoeconomic analysis on TAM and TOR}

Pharmacoeconomics study is to evaluate the cost and effects of a pharmaceutical product. Lin Yan, et al. [11] divided 130 breast cancer patients into TAM group and TOR group according to different therapeutic regimen. The curative efficacy and safety of two therapies were recorded and evaluated using cost-effectiveness analysis in pharmacoeconomics. These results demonstrated that in TAM group vs. TOR group, the drug cost stood at 90.30 yuan vs. 809.52 yuan, with effective rate of $88.75 \%$ vs. $94.00 \%$. In terms of pharmacoeconomics, TAM is superior to TOR. Juhani $U$, et al. [19] refered in the study that the acquisition cost of 1 day's treatment with toremifene was about 3 times higher than that of tamoxifen. However yet little formal pharmacoeconomic analyses comparing TOR with TAM had been published.

\section{Conclusion}

The new generation of Selective Estrogen Receptor Modulators (SERMs) drug TOR can regulate the endogenous estrogen levels in the body. It works as a substitution of endocrine therapy for early stage. It's also friendly to those breast cancer patients who cannot tolerate TAM. Besides, it is used for the treatment of postoperative recurrent and metastatic breast cancer. TOR is a relatively safe and effective drug for breast cancer patients, as is showed in large sample studies that when the usage time reaches 5 years or even longer, OS and DFS of TOR will be much higher than those of TAM. In terms of adverse reactions, especially in the aspects of gynecological safety and ameliorating blood lipid, TOR is better than TAM. Although there have already existed studies which indicate that TAM is better than TOR from the perspective of pharmacoeconomics, those exist studies are not done without limitations. Firstly, studies taken into Metaanalysis do not have well heterogeneity. For example, the dose of TAM and TOR are not similar, the included criteria is not unified, the follow-up year are not the same and the quality of evidence grade of meta analysis is not at a high level. Secondly, it lacks of large sample and multi center randomized controlled studies to confirm the fact. The comparisons between different districts or even different countries are missing. Thirdly, little formal pharmacoeconomic analyses comparing TOR with TAM had been showed in published papers therefore cannot lead to convincing conclusions.

In some cases, the combination use of TOR and TAM can improve the short-term effective cure rate and the quality of life of patients. Overall, compared with the traditional TAM, TOR has a potential security advantage. Larger sample, multi center and randomized con- trolled studies are needed to further investigate the efficacy, safety and pharmacoeconomics of TOR and TAM. Meanwhile, the related mechanisms and differences of the two on the endocrine therapy also require further validation analyses.

\section{References}

1. Chen Wanqing, Zheng Rongshou, Zhang Siwei (2012) An analysis of cancer incidence in china, 2003 2007. China Cancer 21: 161-170.

2. Zhang Lin, Lin Hong (2010) The comparative study of toremifene and tamoxifen on postoperative patients of early breast cancer. Contemporary Medicine 16: 36-37.

3. Qin Tao, Yuan Zhongyu, Peng Roujun, BAI Bing, SHI Yanxia, et al. (2013) Efficacy and safety of toremifene in young patients with luminal subtype early breast cancer: A retrospective study. Journal of Sun Yat-sen University (Medical Sciences) 34: 402-406.

4. Kimura M, Tominaga T, Kimijima I, Takatsuka Y, Takashima S, et al. (2014) Phase III randomized trial of toremifene versus tamoxifen for Japanese postmenopausal patients with early breast cancer. Breast Cancer 21: 275-283.

5. Ma Dachang, Wu Duoming, Xiao Yi (2012) A Meta analysis of toremifene comparison tamoxifen for early stage breast cancer. Journal of Lanzhou University (Medical Sciences) 38: 26-30.

6. Chi F, Wu R, Zeng Y, Xing R, Liu Y, et al. (2013) Effects of toremifene versus tamoxifen on breast cancer patients: a meta-analysis. Breast Cancer 20: 111-122.

7. Lu Qiang (2011) Clinical comparative study of tamoxifen and toremifene in the treatment of recurrence or metastatic breast cancer. Contemporary Medicine 17: 128-129.

8. Zhou WB, Ding Q, Chen L, Liu XA, Wang S (2011) Toremifene is an effective and safe alternative to tamoxifen in adjuvant endocrine therapy for breast cancer: Results of four randomized trials. Breast Cancer Res Treat 128: 625631.

9. Ye QL, Zhai ZM (2014) Toremifene and tamoxifen have similar efficacy in the treatment of patients with breast cancer: a meta-analysis of randomized trials. Mol Biol Rep 41: 751-756.

10. Ma Wen, Wang Xiaohu, Yang Kehu, TIAN Jin-Hui, LIU RuiFeng, et al. (2009) Toremifene and Tamoxifen for Patients with Advanced Breast Cancer: A Meta-analysis of Randomized Controlled Trials. Medical Schools 1026-1029.

11. Lin Yan, Tao Honghui, Yan Chushun, WENG Le-jin, DAI Jia-lin (2012) Pharmacoeconomic Analysis on Tamoxifen vs. Toremifene for Treatment of Breast Cancer. Evaluation and Analysis of Drug-Use in Hospitals of China 12: 330332.

12. Yan Han, Zhang Ruixue, Li Qin (2014) The efficacy and safety of toremifene versus tamoxifen in the treatment of perimenopausal and postmenopausal breast cancer: a system assessment. International Journal of Pathology and Clinical Medicine 34: 713-720.

13. Gershanovich M, Garin A, Baltina, Kurvet A, Kangas LD, et al. (1997) A phase III comparison of two toremifene doses to tamoxifen in postmenopausal women with advanced breast cancer. Eastern European Study Group. Breast Cancer Res Treat 45: 251-262.

14. Hayes DF, Van Zyl JA, Hacking A, Goedhals L, Bezwoda WR, et al. (1995) Randomised comparison of tamoxifen 
and two separate doses of toremifene in postmenopausal patients with metastatic breast cancer. J Clin Oncol 13: 2556-2566.

15. Pagani O, Gelber S, Price K, Price K, Zahrieh D, et al. (2004) Toremifene and tamoxifen are equally effective for early-stage breast cancer: First results of international breast cancer study group trials $12-93$ and 14-93. Ann Oncol 15: 1749-1759.

16. Li Guangming, Duan Baiyun (2016) Clinical effect and quality of life of patients with advanced breast cancer treated with toremifene combined with tamoxifen. Chinese Journal of General Surgery 25: 463-466.
17. Li Wenhua, Yin Ling, Xie Yuyan (2010) Clinical efficacy of toremifene combined with tamoxifen in endocrine therapy for breast cancer. China Modern Doctor 48: 120-121.

18. Lan Ying, Hu Die, He Qin (2017) Safety of toremifene versus tamoxifen in the treatment of breast cancer: A systematic review. China Pharmacy 28: 360-364.

19. Maenpaa JU, Ala Fossi SL (1997) Toremifene in postmenopausal breast cancer. Efficacy, safety and cost. Drugs Aging 11: 261-270. 